\title{
ON BOUNDED FUNCTIONS SATISFYING AVERAGING CONDITIONS. II
}

\author{
BY \\ ROTRAUT GOUBAU CAHILL
}

ABSTRACT. Let $S(f)$ denote the subspace of $L^{\infty}\left(R^{n}\right)$ consisting of those real valued functions $f$ for which

$$
\lim _{r \rightarrow 0} \frac{1}{|B(x, r)|} \int_{B(x, r)} f(y) d y=f(x)
$$

\begin{abstract}
for all $x$ in $R^{n}$ and let $L(f)$ be the subspace of $S(f)$ consisting of the approximately continuous functions. A number of results concerning the existence of functions in $S(f)$ and $L(f)$ with special properties are obtained. The extreme points of the unit balls of both spaces are characterized and it is shown that $L(f)$ is not a dual space. As a preliminary step, it is shown that if $E$ is a $G_{\delta}$ set of measure $O$ in $R^{n}$, then the complement of $E$ can be decomposed into a collection of closed sets in a particularly useful way.
\end{abstract}

Introduction. Let $L_{R}^{\infty}\left(R^{n}\right)$ denote the space of all real valued $L^{\infty}\left(R^{n}\right)$ functions. If $f$ is in $L_{R}^{\infty}\left(R^{n}\right)$ and if $E$ is a measurable subset of $R^{n}$, let $J(f, E)$ denote $\int_{E} f$. For each $f$ in $L_{R}^{\infty}\left(R^{n}\right)$ define:

$$
L(f)=\left\{x \in R^{n} \lim _{r \rightarrow 0}(J(|f-f(x)|, B(x, r)) /|B(x, r)|)=0\right\}
$$

where $B(x, r)=\left\{y \in R^{n}|| y-x \mid<r\right\}$, i.e. $L(f)$ is the Lebesgue set of $f$.

$$
S(f)=\left\{x \in R^{n} \mid \lim _{r \rightarrow 0}(J(f, B(x, r)) /|B(x, r)|)=f(x)\right\} .
$$

Let $S(n, T)$ be the subspace of $L_{R}^{\infty}\left(R^{n}\right)$ consisting of those functions $f$ for which $S(f)=R^{n}$, and let $L(n, T)$ be the subspace of $L_{R}^{\infty}\left(R^{n}\right)$ consisting of those functions for which $L(f)=R^{n}$.

A function $f$ in $L_{R}^{\infty}\left(R^{n}\right)$ is defined to be approximately continuous at $x$ if $x$ is a point of density of $\{y|| f(y)-f(x) \mid<\epsilon\}$ for every $\epsilon>0$. It is easy to see that $L(n, T)$ consists precisely of those functions in $L_{R}^{\infty}\left(R^{n}\right)$ which are approximately continuous at each point of $R^{n}$. An example of a function which is in $S(n, T)$ but not in $L(n, T)$ is the function

Received by the editors March 12, 1974.

AMS (MOS) subject classifications (1970). Primary 41A30, 46A20, 46A90. sure 0 in $R^{n}$.

Key words and phrases. Approximately continuous, extreme points, $G_{\delta}$ sets of mea- 


$$
f(x)= \begin{cases}\sin \left(1 /|x|^{n}\right), & x \neq 0, \\ 0, & x=0 .\end{cases}
$$

The same example shows that $S(n, T)$ is not an algebra, whereas it is readily shown that $L(n, T)$ is an algebra.

In this paper a number of results will be obtained about the existence of functions in $S(n, T)$ and $L(n, T)$ which have special properties. The extreme points of the unit balls of these spaces will also be characterized. In the case of $L(n, T)$ it will be shown that there are only two such extreme points.

The proofs depend primarily on the fact that if $E$ is a $G_{\delta}$ subset of measure 0 contained in $R^{n}$, then $E^{\prime}$, the complement of $E$, can be decomposed in a special way into a collection of closed sets $\left\{\Phi_{\lambda}\right\}_{\lambda>1}$ so that the function $\mu$ defined in $R^{n}$ by

$$
\mu(x)= \begin{cases}0, & x \in E, \\ 1 / \inf _{\lambda}\left\{\lambda \mid x \in \Phi_{\lambda}\right\}, & x \notin E,\end{cases}
$$

is approximately continuous and has a number of other useful properties. It will first be shown how to obtain such a decomposition of $E^{\prime}$. The procedure used generalizes a method developed by Zygmunt Zahorski for obtaining a decomposition of the complement of a $G_{\delta}$ set of measure 0 contained in the open interval $(0,1)[2]$.

The work presented here was done as part of a Ph.D. thesis under the guidance of Professor Lee Rubel of the University of Illinois.

Inverse Zahorski functions.

LEMMA 1. Let $M_{1}$ and $M_{2}$ be two bounded measurable subsets of $R^{n}$ with measures $u_{1}$ and $u_{2}$ respectively. Suppose that $M_{2}$ is a closed subset of $M_{1}$ consisting only of points of density of $M_{1}$. Then for every positive number $p$, there is a closed set $M_{p}$ with $M_{2} \subset M_{p} \subset M_{1}$ satisfying:

(1) Every point of $M_{2}$ is a point of density of $M_{p}$ and every point of $M_{p}$ is a point of density of $M_{1}$.

(2) $\left|M_{p}\right| \geqslant u_{2}+\left(1-2^{-1-p}\right)\left(u_{1}-u_{2}\right)$.

(3) Let $x \in M_{2}$, and let $\epsilon$ be an arbitrary number in $(0,1)$. If $r$ is any positive number for which $\left(\left|M_{1} \cap B(x, r)\right| /|B(x, r)|\right) \geqslant 1-\epsilon$, then

$$
\left(\left|M_{p} \cap B(x, r)\right| /|B(x, r)|\right) \geqslant 1-\epsilon-2^{-m-p+c_{n}}
$$

for every positive integer $m$ for which $r \leqslant 1 / m$, where $c_{n}$ is a constant which depends only on the dimension $n$.

Proof. H. Whitney has shown that since $M_{2}$ is closed, $M_{2}^{\prime}$ is a countable union of closed cubes $Q_{k}$ with disjoint interiors, where these cubes may be chosen so that the following conditions hold: 
(1) $\operatorname{diam} Q_{k} \leqslant \operatorname{dist}\left(Q_{k}, M_{2}\right) \leqslant 4 \operatorname{diam} Q_{k}$.

(2) If $Q_{k}^{*}$ is the cube with the same center as $Q_{k}$ and expanded by a factor $1+\epsilon(0<\epsilon<1 / 4, \epsilon$ fixed $)$, then $Q_{k}^{*}$ is contained in the union of all the cubes which touch $Q_{k}$.

(3) For each cube $Q_{k}$ there are at most $N=(12)^{n}$ cubes which touch $Q_{k}$ [1, pp. 167-169].

A cube $Q_{k}$ will be said to be of class $m, m$ a positive integer, if either $(1 /(m+1))<\operatorname{diam} Q_{k}<(1 / m)$ or $m<\operatorname{diam} Q_{k}<m+1$. If $Q_{k}$ is of class $m$ and if $\left|Q_{k} \cap M_{1}\right|>0$, let $F_{k}$ be a closed subset of $Q_{k} \cap M_{1}$ consisting only of points of density of $M_{1}$, with $\left|F_{k}\right| \geqslant\left|Q_{k} \cap M_{1}\right|\left(1-2^{-m-p}\right)$. Set $M_{p}=$ $M_{2} \cup_{k} F_{k}$.

It will be shown that $M_{p}$ satisfies all the required conditions. First, $M_{p}$ is closed, for if $\left\{q_{m}\right\}_{m>1}$ is a convergent sequence in $M_{p}$, say $q_{m} \rightarrow q$, and if $q \notin M_{2}$, then $q$ is in some cube $Q_{s}$ and some neighborhood of $q$ is contained in $Q_{s}^{*}$. Since $Q_{s}^{*}$ is contained in the union of at most $N$ cubes $Q_{k}$, this neighborhood is contained in the union of at most $N$ cubes. Thus for $m$ sufficiently large, say $m \geqslant M,\left\{q_{m}\right\}_{m>M}$ is contained in at most $N$ of the sets $F_{k}$. Since this union is closed, $q$ is in some $F_{k}$ and hence $M_{p}$ is closed.

By construction, each point of $M_{p}$ is a point of density of $M_{1}$. It will now be shown that each point of $M_{2}$ is a point of density of $M_{p}$. The proof will be such that (3) will be proved simultaneously.

Let $x$ be in $M_{2}$. Let $\epsilon$ be an arbitrary number in $(0,1)$ and let $m$ be an arbitrary positive integer. Since, by assumption, $x$ is a point of density of $M_{1}$, there is a $0<\delta \leqslant 1 / m$ such that for $r \leqslant \delta,\left(\left|B(x, r) \cap M_{1}\right| /|B(x, r)|\right)>1-\epsilon$. Set

$$
d(x, r)=\left(\left|M_{1} \cap B(x, r)\right| /|B(x, r)|\right)-\left(\left|M_{p} \cap B(x, r)\right| /|B(x, r)|\right) .
$$

It will be shown that $d(x, r) \leqslant 2^{-m-p+c_{n}}$. From this it follows that $\left(\left|M_{p} \cap B(x, r)\right| /|B(x, r)|\right)>1-\epsilon-2^{-p+c_{n}-m}$, which verifies (1) since $\epsilon$ and $m$ were arbitrary.

The proof that $d(x, r) \leqslant 2^{-m-p+c_{n}}$ will depend only on the fact that $m$ is a positive integer for which $r<1 / m$. Thus (3) will also be proved.

Let $K$ be the set of all integers for which $Q_{k}$ has nonempty intersection with the boundary of $B(x, r)$ and set

$$
\begin{aligned}
A & =\bigcup_{k \in K} Q_{k} ; A_{1}=A \cap B(x, r) ; A_{2}=A \cap B(x, r)^{\prime} ; \\
\xi & =\left|M_{p} \cap A\right| /\left|M_{1} \cap A\right| \text { if }\left|M_{1} \cap A\right|>0, \quad \xi=1 \text { if }\left|M_{1} \cap A\right|=0 ; \\
\xi_{1} & =\left|M_{p} \cap A_{1}\right| /\left|M_{1} \cap A_{1}\right| \text { if }\left|M_{1} \cap A_{1}\right|>0, \quad \xi_{1}=1 \text { if }\left|M_{1} \cap A_{1}\right|=0 ;
\end{aligned}
$$




$$
\xi_{2}=\left|M_{p} \cap A_{2}\right| /\left|M_{1} \cap A_{2}\right| \text { if }\left|M_{1} \cap A_{2}\right|>0, \quad \xi_{2}=1 \text { if }\left|M_{1} \cap A_{2}\right|=0 .
$$

We have

$$
d(x, r)=(1 /|B(x, r)|)\left\{\sum_{Q_{k} \subset B(x, r)}\left|\left(Q_{k} \cap M_{1}\right)-F_{k}\right|+\left|\left(M_{1}-M_{p}\right) \cap A_{1}\right|\right\} .
$$

Since diam $Q_{k}<\operatorname{dist}\left(Q_{k}, M_{2}\right)<r<1 / m$ for each cube $Q_{k}$ which intersects $B(x, r),\left|\left(Q_{k} \cap M_{1}\right)-F_{k}\right|<2^{-m-p}\left|Q_{k} \cap M_{1}\right|$, and thus

$$
d(x, r) \leqslant(1 /|B(x, r)|)\left\{2^{-m-p} \sum_{Q_{k} \subset B(x, r)}\left|Q_{k} \cap M_{1}\right|+\left|\left(M_{1}-M_{p}\right) \cap A_{1}\right|\right\} .
$$

Thus if $\left|M_{1} \cap A_{1}\right|=0, d(x, r) \leqslant 2^{-m-p}$.

Suppose $\left|M_{1} \cap A_{1}\right|>0$. Observe that

$$
d(x, r) \leqslant(1 /|B(x, r)|)\left\{2^{-m-p} \sum_{Q_{k} \subset B(x, r)}\left|Q_{k} \cap M_{1}\right|+\left(1-\xi_{1}\right)\left|M_{1} \cap A_{1}\right|\right\} .
$$

The object of the calculations which follow is to show that $1-\xi_{1}$ $\leqslant 2^{-m-p}\left\{1+\left(\left|A_{2}\right| /\left|M_{1} \cap A_{1}\right|\right)\right\}$.

By solving the equation $\xi\left|M_{1} \cap A\right|=\xi_{1}\left|M_{1} \cap A_{1}\right|+\xi_{2}\left|M_{1} \cap A_{2}\right|$ for $\xi_{1}$ and observing that $\left|M_{1} \cap A_{2}\right|=\left|M_{1} \cap A_{1}\right|+\left|M_{1} \cap A_{2}\right|$, we obtain

$$
\xi_{1}=\xi-\left(\xi_{2}\left|M_{1} \cap A_{2}\right|-\xi\left|M_{1} \cap A_{2}\right|\right) /\left|M_{1} \cap A_{1}\right| \text {. }
$$

Since $\left|F_{k}\right| \geqslant\left|Q_{k} \cap M_{1}\right|\left(1-2^{-m-p}\right)$ for each $Q_{k}$ which intersects $B(x, r)$, $\left|M_{p} \cap A\right| \geqslant\left(1-2^{-m-p}\right)\left|M_{1} \cap A\right|$ and $\xi>1-2^{-m-p}$. Thus

$$
\xi_{1}>1-2^{-m-p}-\left\{\left(\xi_{2}-\left(1-2^{-m-p}\right)\right) /\left|M_{1} \cap A_{1}\right|\right\}\left|M_{1} \cap A_{2}\right| .
$$

Since $0<\xi_{2} \leqslant 1$ and $\left|M_{1} \cap A_{2}\right| \leqslant\left|A_{2}\right|$,

$$
\xi_{1}>1-2^{-m-p}-2^{-m-p}\left|A_{2}\right| /\left|M_{1} \cap A_{1}\right|,
$$

and

$$
1-\xi_{1}<2^{-m-p}\left\{1+\left(\left|A_{2}\right| /\left|M_{1} \cap A_{1}\right|\right)\right\}
$$

It follows that

$$
\begin{aligned}
d(x, r) & \leqslant 2^{-m-p}\left\{1+\left\{\left|M_{1} \cap A_{1}\right|\left(1+\left(\left|A_{2}\right| /\left|M_{1} \cap A_{1}\right|\right)\right)\right\} /|B(x, r)|\right\} \\
& \leqslant 2^{-m-p}\left\{2+\left(\left|A_{2}\right| /|B(x, r)|\right)\right\} .
\end{aligned}
$$

Since diam $Q_{k} \leqslant r$ for each $Q_{k}$ which intersects $B(x, r), A_{2} \subset B(x, 2 r)-$ $B(x, r)$. Thus,

$$
\left(\left|A_{2}\right| /|B(x, r)|\right) \leqslant(1 /|B(x, r)|)(|B(x, 2 r)|-|B(x, r)|) .
$$


The number $d_{n}=(1 /|B(x, r)|)(|B(x, 2 r)|-|B(x, r)|)$ depends only on $n$ and $d(x, r) \leqslant 2^{-m-p}\left(2+d_{n}\right) \leqslant 2^{-m-p+c_{n}}$, where $2^{c_{n}}=2+d_{n}$. Therefore, (1) and (3) both hold.

Finally,

$$
\begin{aligned}
\left|M_{p}\right| & =\left|M_{2}\right|+\sum_{k}\left|F_{k}\right| \geqslant u_{2}+\sum_{k}\left|Q_{k} \cap M_{1}\right|\left(1-2^{-m-p}\right) \\
& >u_{2}+\left(1-2^{-1-p}\right) \sum_{k}\left|Q_{k} \cap M_{1}\right|=u_{2}+\left(1-2^{-1-p}\right)\left(u_{1}-u_{2}\right),
\end{aligned}
$$

so that (2) also holds. Q.E.D.

COROLLARY 1. For each $G_{\delta}$ set $E$, of measure 0 in $R^{n}$, there is an increasing sequence of compact sets $\left\{F_{k}\right\}_{k>1}$ with $\left|\Phi_{k}\right|>k$ such that $E^{\prime}=$ $\bigcup_{k} \Phi_{k}$ and $\left|B(x, r) \cap \Phi_{k+1}\right| /|B(x, r)|>1-2^{-m-k+c_{n}}$ whenever $x \in \Phi_{k}$ and $r \leqslant 1 / m, m$ a positive integer.

Proof. Since $E$ is a $G_{\delta}$ of measure 0 , there exists an increasing sequence of closed sets $\left\{F_{k}\right\}_{k>1}$ with $E^{\prime}=\bigcup_{k>1} F_{k}$. Let $\left\{a_{k}\right\}_{k>1}$ be a strictly increasing sequence of positive numbers for which $\left|B\left(0, a_{k}\right)\right|>\left(1 /\left(1-2^{-k}\right)\right) \cdot$ $\left(k-2^{-k}\right)$ and for which $a_{k+1}-a_{k}$ is greater than 1 for all $k$. Let $P_{1}$ be any closed subset of $E^{\prime} \cap B\left(0, a_{1}\right)$ for which $\left|P_{1}\right|>1$ and set $\Phi_{1}=P_{1} \cup\left(F_{1} \cap\right.$ $\left.B\left(0, a_{1}\right)\right)$.

Since $\Phi_{1} \subset E^{\prime} \cap B\left(0, a_{2}\right)$ and $\left|\Phi_{1}\right|+\left(1-2^{-2}\right)\left(\left|B\left(0, a_{2}\right)\right|-\left|\Phi_{1}\right|\right)>2$, the preceding lemma implies that there is a closed set $P_{2}$ of measure greater than 2 with $\Phi_{1} \subset P_{2} \subset E^{\prime} \cap B\left(0, a_{2}\right)$ which satisfies conditions (1), (2) and (3) of the lemma, with $M_{2}=\Phi_{1}, M_{1}=E^{\prime} \cap B\left(0, a_{2}\right)$ and $p=1$.

For each $x$ in $\Phi_{1}$ and $r<1, B(x, r) \subset B\left(0, a_{2}\right)$ since $a_{2}-a_{1}>1$. Thus $\left|E^{\prime} \cap B\left(0, a_{2}\right) \cap B(x, r)\right| /|B(x, r)|=1$ and by (3) of Lemma 4.1,

$$
\left|P_{2} \cap B(x, r)\right| /|B(x, r)| \geqslant 1-2^{-m-1+c_{n}}
$$

for every positive integer $m$ such that $r \leqslant 1 / m$. Set $\Phi_{2}=P_{2} \cup\left(F_{2} \cap B\left(0, a_{2}\right)\right)$.

Continue inductively. Having defined $\Phi_{k}$ for $k \leqslant s$ so that $\Phi_{k} \subset F_{k} \cap$ $B\left(0, a_{k}\right),\left|\Phi_{k}\right|>k$ and $\left|\Phi_{k} \cap B(x, r)\right| /|B(x, r)|>1-2^{-m-(k-1)+c_{n}}$ for $x \in$ $\Phi_{k-1}$ and $r \leqslant 1 / m$, let $P_{s+1}$ be a closed set of measure greater than $s+1$ for which $\Phi_{s} \subset P_{s+1} \subset E^{\prime} \cap B\left(0, a_{s+1}\right)$ and for which (1), (2) and (3) of Lemma 4.1 hold with $M_{2}=\Phi_{s}, p=s$ and $M_{1}=E^{\prime} \cap B\left(0, a_{s+1}\right)$. Since $\left|E^{\prime} \cap B\left(0, a_{s+1}\right) \cap B(x, r)\right| /|B(x, r)|$ equals 1 for each $x$ in $\Phi_{s}$ and $r<1$, (3) of the lemma implies that $\left|P_{s+1} \cap B(x, r)\right| /|B(x, r)|>1-2^{-s-m+c_{n}}$ for each positive integer $m$ for which $r<1 / m$. Set $\Phi_{s+1}=P_{s+1} \cup\left(F_{s+1} \cap\right.$ $\left.B\left(0, a_{s+1}\right)\right)$.

The sequence $\left\{\Phi_{k}\right\}_{k>1}$ satisfies the conditions of the theorem. Q.E.D.

We observe that by suitable choice of $a_{1}$ and $P_{1}, \Phi_{1}$ can be made to contain a specified compact subset of $E^{\prime}$. 
If $E$ is a $G_{\delta}$ set of measure 0 in $R^{n}$, an increasing sequence of compact subsets of $R^{n}$ satisfying the conditions of Corollary 1 will be called a Zahorski sequence for $E$.

THEOREM 1. Let $E$ be $a G_{\delta}$ set of measure 0 in $R^{n}$. There exists a real valued, measurable function $u$ defined on $R^{n}$ having the following properties:

(1) $0 \leqslant u \leqslant 1$.

(2) $u$ is 0 precisely on $E$.

(3) $u$ is continuous at each point of $E$.

(4) For every $x_{0}$ in $R^{n}$ and every $\epsilon>0$, there is an $r>0$ such that $u(x)$ $\leqslant(1 /(1-\epsilon)) u\left(x_{0}\right)$ whenever $x$ is in $B\left(x_{0}, r\right)$.

(5) Every $x$ in $R^{n}$ is a Lebesgue point of $u$.

Proof. Let $\left\{\Phi_{k}\right\}_{k \geqslant 1}$ be a Zahorski sequence for $E$. A closed set $\Phi_{r}$ will now be defined for each number $r$ of the form $m / 2^{s}$, where $m$ and $s$ are positive integers and $m>2^{s}$. These closed sets will satisfy these two conditions:

(a) $\Phi_{s^{\prime}} \subset \Phi_{s}$ if $s>s^{\prime}$,

(b) $\Phi_{s^{\prime}}$ consists only of points of density of $\Phi_{s}$ if $s>s^{\prime}$.

For each odd integer $k>2, k=2 m+1$, let $\Phi_{k / 2}$ be a closed set with $\Phi_{m} \subset \Phi_{k / 2} \subset \Phi_{m+1}$ and $\left|\Phi_{k / 2}\right|>1 / 2\left(\left|\Phi_{m+1}\right|+\left|\Phi_{m}\right|\right)$, for which every point of $\Phi_{m}$ is a point of density of $\Phi_{k / 2}$ and every point of $\Phi_{k / 2}$ is a point of density of $\Phi_{m+1}$. Such a set exists by Lemma 1. Having defined $\Phi_{r / 2}$ for all $r>$ $2^{k}$ and all $k \leqslant s$, let $\Phi_{r / 2} s+1, r>2^{s+1}, r=2 t+1$, be a closed set with

$$
\Phi_{t / 2 s} \subset \Phi_{r / 2 s+1} \subset \Phi_{(t+1) / 2^{s}} \text { and }\left|\Phi_{r / 2 s+1}\right|>1 / 2\left(\left|\Phi_{(t+1) / 2^{s}}\right|\right) \text {, }
$$

for which each point of $\Phi_{t / 2}$ is a point of density of $\Phi_{r / 2 s+1}$ and each point of $\Phi_{r / 2^{s+1}}$ is a point of density of $\Phi_{(t+1) / 2^{s}}$.

Now let $\lambda$ be any real number greater than or equal to 1 and défine $\Phi_{\lambda}=$ $\bigcap_{m>\lambda 2^{k}} \Phi_{m / 2^{k}}$. The collection of closed sets $\left\{\Phi_{\lambda}\right\}_{\lambda>1}$ also satisfies (a) and (b).

Define the function $u$ on $R^{n}$ by

$$
u(p)= \begin{cases}1 / \inf \left\{\lambda \mid p \in \Phi_{\lambda}\right\} & \text { if } p \notin E, \\ 0 & \text { if } p \in E .\end{cases}
$$

Properties (1) and (2) from the statement of the theorem follow immediately from the definition of $u$. (3)-(5) will now be verified.

Let $p$ be in $E$, and let $\epsilon>0$ be arbitrary. If $r$ is less than $\operatorname{dist}\left(p, \Phi_{1 / \epsilon}\right)$, then $B(p, r) \cap \Phi_{1 / \epsilon}$ is empty and $u(x)$ is less than $\epsilon$ for $x$ in $B(p, r)$. Thus $u$ is continuous on $E$.

Let $x_{0}$ be in $E^{\prime}$, and let $\epsilon>0$ be arbitrary. If $r$ is less than $\operatorname{dist}\left(x_{0}, \Phi_{(1-\epsilon) / u\left(x_{0}\right)}\right)$, then $u(x) \leqslant u\left(x_{0}\right) /(1-\epsilon)$ for all $x$ in $B\left(x_{0}, r\right)$. Thus (4) holds. This property ensures that $u$ is measurable. 
Since $u$ is continuous on $E$, (5) holds for every $x$ in $E$. Let $x_{0}$ be in $E^{\prime}$ and let $\epsilon>0$ be arbitrary. Since $x_{0}$ is in $\Phi_{(1+\epsilon / 2) / u\left(x_{0}\right)}, x_{0}$ is a point of density of $\Phi_{(1+\epsilon) / u\left(x_{0}\right)}$ and thus of $\left\{y \mid u(y) \geqslant u\left(x_{0}\right) /(1+\epsilon)\right\}$. This, together with (4) and the boundedness of $u$, yields (5).

Thus $u$ satisfies all the required conditions. Q.E.D.

If $E$ is a $G_{\delta}$ set of measure 0 in $R^{n}$, a collection of closed sets $\left\{\Phi_{\lambda}\right\}_{\lambda>1}$, constructed in the manner of the first part of the proof of this last theorem, will be called a Zahorski collection for $E$. The function

$$
u(x)= \begin{cases}1 / \inf _{\lambda}\left\{\lambda \mid x \in \Phi_{\lambda}\right\}, & x \notin E, \\ 0, & x \in E,\end{cases}
$$

will be called the corresponding inverse Zahorski function.

Applications to $S(n, T)$ and $L(n, T)$. An immediate consequence of Theorem 1 is

THEOREM 2. If $E$ is $a G_{\delta}$ set of measure 0 in $R^{n}$, then there is a function in $L(n, T)$ of norm 1 which vanishes precisely on $E$.

Proof. Let $u$ be an inverse Zahorski function for $E$. $u$ has norm 1 and vanishes precisely on $E$. Since, in addition, every point of $R^{n}$ is a Lebesgue point of $u, u$ satisfies the conditions of the theorem. Q.E.D.

If $E$ is a $G_{\delta}$ of measure 0 contained in $R^{n}$ and if $F$ is a compact subset of $E^{\prime}$, then it is possible to find a Zahorski collection $\left\{\Phi_{\lambda}\right\}_{\lambda>1}$ for $E$ for which $F$ is a subset of $\Phi_{1}$. The corresponding inverse Zahorski function has norm 1 , is 0 on $E$ and identically 1 on $F$. Since every point of $R^{n}$ is a Lebesgue point of $u, u$ is in $L(n, T)$. We therefore also have

THEOREM 3. If $E$ is a $G_{\delta}$ of measure 0 in $R^{n}$ and if $F$ is a compact subset of $R^{n}$, disjoint from $E$, then there is a function of norm 1 in $L(n, T)$ which is 0 at each point of $E$ and 1 at each point of $F$.

COROLlaRY 2. If $\left\{w_{k}\right\}_{k>1}$ is an arbitrary sequence of distinct points in $R^{n}$ and if $\left\{a_{k}\right\}_{k \geqslant 1}$ is an absolutely summable sequence of real numbers, then there is a function $g$ in $L(n, T)$ for which $g\left(w_{k}\right)=a_{k}$ for all $k$.

Proof. For each $i$, let $S_{i}$ be a $G_{\delta}$ of measure 0 containing $\left\{w_{k}\right\}_{k>1}-$ $\left\{w_{i}\right\}$ and not containing $w_{i}$. Let $u_{i}$ be an inverse Zahorski function for $S_{i}$ for which $u_{i}\left(w_{i}\right)=1$.

Since $\Sigma_{k=1}^{\infty}\left|a_{k}\right|<\infty$ and $\left\|u_{i}\right\|_{\infty}=1$ for all $i$, every point of $R^{n}$ is a Lebesgue point of the function $g=\sum_{k=1}^{\infty} a_{k} u_{k}$. Thus $g$ is in $L(n, T)$. Since $u_{i}\left(w_{k}\right)=\delta_{i k}, g\left(w_{k}\right)=a_{k}$ for every $k$. Q.E.D.

COROLlaRY 3. If $\left\{w_{k}\right\}_{k \geqslant 1}$ is a convergent sequence of distinct points of 
$R^{n}$ with limit $w \neq w_{k}$ any $k$ and if $\left\{a_{k}\right\}_{k \geq 1}$ is an arbitrary sequence of 0 's and 1 's, then there is a function $g$ in $L(n, T)$, with $\|g\|_{\infty}=1$, for which $g\left(w_{k}\right)=a_{k}$ for all $k$.

The proof is similar to that of Corollary 2.

LEMMA 2. Let $f$ be in $L_{R}^{\infty}\left(R^{n}\right)$ and let $E$ be a $G_{\delta}$ of measure 0 containing $\{x \mid x \notin L(f)\}$. If $u$ is an inverse Zahorski function for $E$, then uf is in $L(n, T)$.

Proof. It is sufficient to show that $L(u f)=R^{n}$. If $x$ is in $E, u(x)=0$ and

$$
\lim _{r \rightarrow 0} J(|u f-u(x) f(x)|, B(x, r)) /|B(x, r)|=\lim _{r \rightarrow 0} J(|u|, B(x, r)) /|B(x, r)|=0 .
$$

If $x \notin E$, then $x$ is a Lebesgue point of both $u$ and $f$ and so also for the product. Q.E.D.

Thus every function in $L_{R}^{\infty}\left(R^{n}\right)$ can be multiplied by a suitable inverse $\mathrm{Za}$ horski function so that the product is in $L(n, T)$.

THEOREM 4. If $f$ is in $L_{R}^{\infty}\left(R^{n}\right)$ and if $F$ is a compact subset of the Lebesgue points of $f$, then there is a function in $L(n, T)$ whose restriction to $F$ is $f$.

Proof. Let $E$ be a $G_{\delta}$ of measure 0 disjoint from $F$, which contains $\left\{x \in R^{n} \mid x \notin L(f)\right\}$. Let $\left\{\Phi_{\lambda}\right\}_{\lambda>1}$ be a Zahorski collection for $E$ with $F \subset \Phi_{1}$ and let $u$ be the corresponding inverse Zahorski function. $u f$ is the required function. Q.E.D.

Consequently $L(n, T)$ is locally dense in measure in $L_{R}^{\infty}\left(R^{n}\right)$, i.e. if $F$ is a compact subset of $R^{n}$, then there is a sequence of functions in $L(n, T)$ which converges in measure to $f$ on $F$.

Lemma 2 may be applied to characterize the extreme points of the unit ball of $S(n, T)$.

THEOREM 5. $F$ is an extreme point of the unit ball of $S(n, T)$ if and only if $|F|=1$ a.e.

Proof. If $|F|=1$ a.e., then $F$ is an extreme point of the unit ball of $L_{R}^{\infty}\left(R^{n}\right)$ and hence also of $S(n, T)$. Conversely, suppose $F$ fails to have modulus 1 at each point of some subset of $R^{n}$ of positive measure. Let $E$ be a $G_{\delta}$ of measure 0 in $R^{n}$ containing $\left\{x \in R^{n} \mid x \notin L(1-|F|)\right\}$. Let $u$ be an inverse Zahorski function for $E$. By Lemma 5.1, $u(1-|F|)$ is in $L(n, T)$ and so in $S(n, T)$. Since $u(1-|F|) \leqslant 1-|F|,\|u(1-|F|)-F\|_{\infty} \leqslant 1$ and $\|u(1-|F|)+F\|_{\infty} \leqslant 1$ so that $F$ is not extreme. Q.E.D.

It is easy to see that the same result holds for the unit ball of $L(n, T)$, i.e. $F$ is an extreme point of the unit ball of $L(n, T)$ if and only if $|F|=1$ a.e. If 
$|F|=1$ a.e., then $F$ is an extreme point of $S(n, T)$ and so also of $L(n, T)$. If $F$ is in $L(n, T)$, then it follows from the inequality

$$
J(|| F|-| F(x)||, B(x, r)) \leqslant J(|F-F(x)|, B(x, r))
$$

that $1-|F|$ is also in $L(n, T)$. Thus if $|F|$ is less than 1 on a set of positive measure, then $G=1-|F|$ is a function in $L(n, T)$ which satisfies $\|F-G\|_{\infty} \leqslant$ 1 and $\|F+G\|_{\infty} \leqslant 1$ so that $F$ is not extreme.

THEOREM 6. $L(n, T)$ is not the dual of a Banach space.

Proof. It is sufficient to show that the only extreme points of the unit ball of $L(n, T)$ are the constant functions 1 and -1 . That this is so is a consequence of the following lemma:

LEMmA 3. If $f$ is a function in $L(n, T)$ which assumes the value 0 or 1 a.e., then $f$ is constant.

PRoOF. Let $g(x)=f(x)(1-f(x))$. Since

$$
g(x)=\lim _{r \rightarrow 0} J(g, B(x, r)) /|B(x, r)|=0
$$

for each $x$ in $R^{n}, f$ actually assumes the values 0 or 1 everywhere.

Let $K=\left\{x \in R^{n} \mid f\right.$ is discontinuous at $\left.x\right\}$. It is sufficient to show that $K$ is empty.

Suppose $K$ is not empty.

Claim. If $x_{0} \in K$, then every neighborhood of $x_{0}$ contains some $x$ in $K$ for which $f(x) \neq f\left(x_{0}\right)$.

Proof OF Claim. Let $x_{0}$ be in $K$ and suppose, without loss of generality, that $f\left(x_{0}\right)=1$. Let $B\left(x_{0}, r\right)$ be an arbitrary ball in $R^{n}$ with center at $x_{0}$ and having radius $r$. Let $s$ be any number in $(0, r / 2)$. Since $f$ is discontinuous at $x_{0}$, there is some $a$ in $B\left(x_{0}, s\right)$ for which $f(a)=0$. If $a$ is in $K$, we are done. If $a$ is not in $K, f$ is continuous at $a$ and so vanishes in a neighborhood of $a$. Set $t_{a}=\sup _{t}\{t>0 \mid f$ is identically 0 in $B(a, t)\} . B\left(a, t_{a}\right)$ is a subset of $B\left(x_{0}, r\right)$ and is not tangent to $B\left(x_{0}, r\right)$ at any point. (Otherwise we would have $x_{0}$ in $B\left(a, t_{a}\right)$ but $f\left(x_{0}\right)=1$.) Let $x$ be an arbitrary point on the boundary of $B\left(a, t_{a}\right)$. We have

$$
\begin{aligned}
f(x) & =\lim _{r \rightarrow 0} J(f, B(x, r)) /|B(x, r)| \\
& =\lim _{r \rightarrow 0} J\left(f, B(x, r) \cap B\left(a, t_{a}\right)^{\prime}\right) /|B(x, r)| \\
& <\lim _{r \rightarrow 0}\left|B(x, r) \cap B\left(a, t_{a}\right)^{\prime}\right| /|B(x, r)|<1 .
\end{aligned}
$$

Thus $f(x)=0$ and $f$ vanishes on the boundary of $B\left(a, t_{a}\right)$. By choice of $t_{a}$ and 
compactness of the boundary, $f$ must have at least one discontinuity $x^{\prime}$ on the boundary of $B\left(a, t_{a}\right)$. Since $x^{\prime}$ is in $K \cap B\left(x_{0}, r\right)$ and $f\left(x^{\prime}\right) \neq f\left(x_{0}\right)$, the proof of the claim is complete.

Now let $x_{1}$ be in $K$ with $f\left(x_{1}\right)=1$ and let $0<r_{1}<1 / 2$ be such that for $0<r<r_{1}, J\left(f, B\left(x_{1}, r\right)\right) /\left|B\left(x_{1}, r\right)\right|>1-1 / 2$.

Let $x_{2}$ be any point in $K \cap B\left(x_{1}, r_{1}\right)$ for which $f\left(x_{2}\right)=0$, and let $0<$ $r_{2}<1 / 2{ }^{2}$ be such that for $0<r<r_{2}, J\left(f, B\left(x_{2}, r\right)\right) /\left|B\left(x_{2}, r\right)\right|<1 / 2$ and $\bar{B}\left(x_{2}, r_{2}\right) \subset B\left(x_{1}, r_{1}\right)$.

Continue defining $x_{k}$ and $r_{k}$ inductively as follows: If $k$ is odd, let $x_{k}$ be any point in $K \cap B\left(x_{k-1}, r_{k-1}\right)$ for which $f\left(x_{k}\right)=1$ and let $0<r_{k}<12^{k}$ be such that for $0<r<r_{k}, \bar{B}\left(x_{k}, r\right) \subset B\left(x_{k-1}, r_{k-1}\right)$ and $J\left(f, B\left(x_{k}, r\right)\right) /\left|B\left(x_{k}, r\right)\right|$ $>1-12^{k}$. If $k$ is even choose $x_{k}$ and $r_{k}$ in a similar way except that $f\left(x_{k}\right)=0$ and $J\left(f, B\left(x_{k}, r\right)\right) /\left|B\left(x_{k}, r\right)\right|<12^{k}$ for $0<r<r_{k}$. Let $x$ be in the intersection of the $\bar{B}\left(x_{k}, r_{k}\right)$. Then

$$
\begin{aligned}
\varlimsup_{k \rightarrow \infty} \mid J(f- & \left.f(x), B\left(x_{k}, r_{k}\right)\right)|/| B\left(x_{k}, r_{k}\right) \mid \\
\leqslant & \left\{\left|B\left(x, 2 r_{k}\right)\right| /\left|B\left(x_{k}, r_{k}\right)\right|\right\} \\
& \times \lim _{k \rightarrow \infty} J\left(|f-f(x)|, B\left(x, 2 r_{k}\right)\right) /\left|B\left(x, 2 r_{k}\right)\right|=0 .
\end{aligned}
$$

But this implies that $f(x)$ must be both 0 and 1 which is impossible. Q.E.D. The example

$$
f(x)=\left\{\begin{aligned}
1 & \text { if } x>0 \\
0 & \text { if } x=0 \\
-1 & \text { if } x<0
\end{aligned}\right.
$$

shows that there are nonconstant extreme points of the unit ball of $S(n, T)$.

\section{REFERENCES}

1. E. M. Stein, Singular integrals and differentiabllity properties of functions, Princeton Univ. Press, Princeton, N. J., 1970. MR 44 \#7280.

2. Z. Zahorski, Über die Menge der Punkte in welchen die Ableitung unendlich ist, Tôhoku Math. J. 48 (1941), 321-330. MR 10, 359.

DEPARTMENT OF MATHEMATICS, UNIVERSITY OF WISCONSIN, WASHINGTON COUNTY CENTER, WEST BEND, WISCONSIN 53095 